\title{
OPEN SOURCE HBIM FOR CULTURAL HERITAGE: A PROJECT PROPOSAL
}

\author{
F. Diara ${ }^{a *}$, F. Rinaudo ${ }^{a}$ \\ ${ }^{a}$ DAD, Department of Architecture and Design, Politecnico di Torino, Viale Mattioli 39, 10129 Torino, Italy \\ filippo.diara@polito.it, fulvio.rinaudo@polito.it
}

Commission II, WG8

KEY WORDS: Accessibility, Cultural Heritage, Dynamic custom-made tool, HBIM, Open source, Usability

\begin{abstract}
:
Actual technologies are changing Cultural Heritage research, analysis, conservation and development ways, allowing new innovative approaches. The possibility of integrating Cultural Heritage data, like archaeological information, inside a three-dimensional environment system (like a Building Information Modelling) involve huge benefits for its management, monitoring and valorisation. Nowadays there are many commercial BIM solutions. However, these tools are thought and developed mostly for architecture design or technical installations. An example of better solution could be a dynamic and open platform that might consider Cultural Heritage needs as priority. Suitable solution for better and complete data usability and accessibility could be guaranteed by open source protocols. This choice would allow adapting software to Cultural Heritage needs and not the opposite, thus avoiding methodological stretches.

This work will focus exactly on analysis and experimentations about specific characteristics of these kind of open source software (DBMS, CAD, Servers) applied to a Cultural Heritage example, in order to verifying their flexibility, reliability and then creating a dynamic HBIM open source prototype. Indeed, it might be a starting point for a future creation of a complete HBIM open source solution that we could adapt to others Cultural Heritage researches and analysis.
\end{abstract}

\section{INTRODUCTION}

The storage and management of the data useful to document a Cultural Heritage asset can be carry out through the utilisation of DBMS (DataBase Management System) and CAD platforms. In Cultural Heritage documentation and management, Building Information Modelling (BIM) extends the possibilities of a traditional CAD capability (Logothetis et al., 2016), allowing to establish geometric and semantic relations between $3 \mathrm{D}$ objects (surveyed as they are) and external data. Indeed, one of the most important capabilities of BIM technology is to combine the geometric information with data of different nature.

However, this solution is not yet adopted at professional level as a standard approach, due to the problems arising from the use of commercial BIM platforms, mainly designed and conceived to support building design or management.

The complexity of the Cultural Heritage assets (e.g. historical buildings, archaeological sites, movable objects, etc.), bring specialists to reflect on the need to create a custom-made instrument, able to include any kind of information and to collect them inside a $3 \mathrm{D}$ environment (or 4D according with time dimension). The aim is to obtain a dynamic tool able to store and manage Cultural Heritage data to allow a correct understanding, a secure archiving and the possibility to inquire data coming from different analysis and expertise.

BIM of existing buildings and facilities (as-built BIM) is still a challenge, especially for the complexity of geometric and not geometric data and its interpretation inside a BIM platform (Barazzetti, 2016).

In this regard open source software may be a fitting choice thanks to its flexibility and customization, allowing new possibilities of integration and classification of features and facilities that cannot directly linked to a classical BIM solution. Innovative and authentic open source BIM platforms, with DBMS, viewers and webservers solutions, could allow overcoming these problems and open the floor to a wide adoption of this technology for Cultural Heritage documentation and management.

It must be noticed that the same phenomenon influenced the research efforts in the past years about the use of GIS technology for Cultural Heritage documentation and management. After a period where researchers try to use commercial GIS platforms, today the first usable platforms based on open source realisations are indicated as the best examples to be used when 2D geometry can be accepted (i.e. management of archaeological excavations, location of objects, monitoring of wide areas, etc.) (Myers, 2016).

In the following paragraphs the analysis and experimentations about specific characteristics of open source software (3D CAD, DBMS, web servers) applied to a Cultural Heritage example are described. The main aim is to verify their flexibility, reliability and then to propose a possible dynamic HBIM (Heritage or Historical BIM) open source system.

\section{FOSS HBIM PROJECT}

The use of open source and free services could be considered a challenge: could Cultural Heritage researches carry out with the integration of low cost instruments and FOSS software? The answer is not obvious, but the experiences gathered on GIS development inside Cultural Heritage documentation allows a reasonable optimism. A suitable solution to guarantee the best and complete data usability and accessibility could be promised by open source protocols, both for software and file formats. This choice would allow to adapt software and DBMS to the Cultural Heritage needs and not the opposite, thus avoiding methodological stretches. In addition, in the Cultural Heritage field (like archaeology), many analysis and researches carried out using FOSS instruments and software have often proved to be most reliable, allowing to obtain more complete results than other researches. 
By using commercial BIM software, (e.g. an historical building or an archaeology project), could be very easy to make compromises, also methodological ones.

The creation of an HBIM of existing buildings requires some precautions, mainly as far as definition and classification of macro and micro building work evidences, single elements and architectural components, database of materials are concerned. Moreover, most of these commercial platforms are in most cases expensive and not suitable for all Cultural Heritage projects (Logothetis et al, March 2017), where the main economic effort must be concentrated on data acquisition and digitisation

The adoption of an open source approach is also a milestone in data preservation and fruition, while closed format are prone to continuous upgrades by the owners and, by considering long periods, can disappear and loose the portability on other platforms.

Despite commercial solutions, open source protocols and software allow to operate directly on source code, allowing modifying, improving, and adapting any tool to specific needs. Inside the project proposal here presented, the adoption of FreeCAD and others open source BIM software follow exactly this thought.

\section{FREECAD CUSTOMIZATION FOR CULTURAL HERITAGE}

FreeCAD is a parametric CAD and BIM open source software based on the presence of different workbenches that allow increasing standard package of FreeCAD and therefore its potentialities. This software is conceived as a 3D parametric modeller: all objects that can be generated have geometries and shapes that depend on own properties or derived from others correlated objects.

Being an open source software, FreeCAD is almost bare from a lot of necessary tools and it requires to be compiled with libraries and packages connected easily inside the software.

\subsection{Assembling FreeCAD}

FreeCAD's development is based on Phyton language and its implementations are available for Windows, MacOS and Linux operating systems users. The use and customization require different open source packages and tools, such as Phyton Installer, PIP (package manager for python), CMake (an opensource, cross-platform family of tools designed to build, test and package software), Homebrew package and XCode (only for $\mathrm{MacOS}$ ).

As far as file format is concerned, FreeCAD manages OBJ file (one of the most common file format of 3D models).

To develop HBIM projects tools as:

- IfcOpenShell (a fundamental open source library to manage IFC file) become necessary being IFC format the starting point of a BIM environment;

- LibLAS and libLAS repository to manage points clouds with LAS file format;

- Graphviz for the visualization of dependencies graphics inside FreeCAD

- PyCollada to import and export 3D models with DAE (Collada) file format.

\subsection{Workbenches customization for Cultural Heritage}

Inside FreeCAD the modification or creation of workbenches (programmed and developed in Python environment), allow the customization of specific commands, tools, roles and definitions depending on the project needs.
To develop an HBIM of an historic building, Arch, Draft and Part Design workbenches are probably the most important modules to be used. Arch workbench has many tools to define all architectural elements of a building, such as wall, structure, and component.

The roles of these elements can be extended and integrated following architecture needs: wall tool can be extended with roles as cladding, buttress, terrace, retaining wall; structure tool could include roles as hole (wall cavity), door, window, loadbearing structure, architrave; component tool could include roles as quoin, brick, roof tile. This database of roles can be extended also regarding archaeological sites.

Is a real challenge to create a custom made workbench (rewriting for instance the Arch workbench) thought for historical buildings or archaeological sites. In this regard, for example, a tool as "stratigraphic units" could be included, based on the stratigraphic evidences survey and interpretation, as parametric surfaces linked to the IFC model.

Moreover, this kind of tool could allow the classification of these units as the archaeological evidences: positive unit (like accumulation activities), negative unit (like erosion or removing activities), neutral unit (like transformation activities), and hidden unit (hidden units behind or under other units).

Consequently, building up an IFC model following these classifications, the recording of the stratigraphic evolution of the historical object is possible.

\subsection{Materials customization for Cultural Heritage}

FreeCAD platform allows to work with a customizable materials database, created specifically for own needs.

This database is referred to the MasterFormat classification (www.buildsite.com/masterformat), used widely for constructions and architectural design inside the most common BIM platforms.

This kind of classification allows the integration of mechanical properties (Ultimate Tensile Strength, Compressive Strength, Youngs Modulus, Elasticity, Fracture Toughness) and architectural properties (Environmental Efficiency Class, Fire Resistance Class, Sound Transmission Class, Thermal Conductivity) of any materials directly corresponding to $3 \mathrm{D}$ objects.

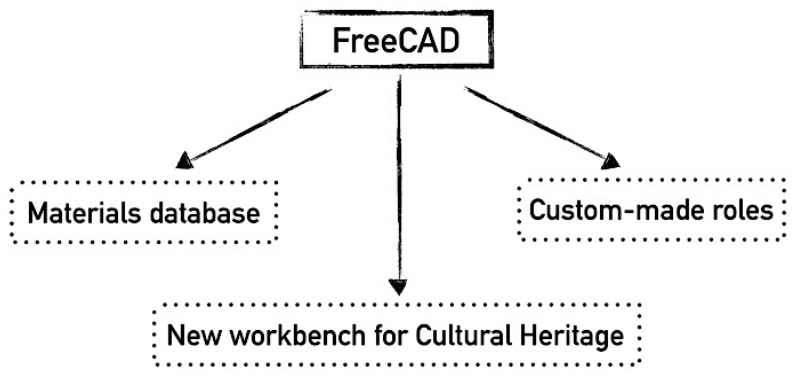

Figure 1. Possible customizations under FreeCAD for the Cultural Heritage projects

The default materials database of FreeCAD contains generic construction materials, such as steel, generic wood, concrete, glass, plastic; these materials are obviously classified for architectural design and engineering projects and are not suitable for historical architecture or archaeological sites.

In order to carry out an HBIM, the customization of this materials database becomes necessary, and FreeCAD allows this kind of intervention.

By using the materials editor tool the creation of a custommade material library becomes possible (for example new_material.FCMat): starting from MasterFormat, the new 
classification can be included inside the material card by specifying information and properties such as mechanical, architectural, rendering and generic properties.

In this way, an HBIM project carried out with FreeCAD could integrates a custom-made materials database (e.g. travertine, marble, limestone, sandstone, dirt, brick, mortar, plaster, iron, bronze and other materials)

\section{CREATE AND MODIFY A CULTURAL HERITAGE IFC MODEL}

Inside an HBIM project, IFC models have a fundamental importance

IFC (Industry Foundation Classes) open format, developed by "buildingSMART" and used by the AEC industry (Architecture, Engineering and Construction), is referred to a relational object characterized by different patterns and layers that includes different classes.

IFC main features are defined with the EXPRESS language (ISO/TC 184/SC 4.1994) and these are: header section, types, entities, functions, and rules.

The header section gives generic information about the schema; types and entities are directly related to the data definitions; functions and rules define the constraints imposed on data definitions (Ghang et al., 2014).

An IFC model could considered as a dynamic database: inside it, it is possible to include a lot of information, such as the object's material, the physical relationships with other models, and the mechanical properties.

Beyond geometry, elements and surfaces extracted from point clouds must be assigned to classes of objects and typological sub divisions (Macher et al, 2017). In this sense an extruded surface from point clouds become an IFC parametric wall entity or an architectural structure (based on real volumes) with specific properties and materials.

Using a parametric BIM/CAD software, such as FreeCAD, it is possible to define geometric relationships between elements, (e.g. according to the real situation of the architectural composition)

The basic structure of an IFC parametric model is based on parametric object, that briefly contain geometric information and associated data and rules (Barazzetti, 2016).

To create a parametric model of existing Cultural Heritage objects, the metric acquisition and processing phase represents the first step, and it can be carry out by using different techniques, such as LiDAR or photogrammetric approach (e.g. terrestrial, and UAV). The obtained point clouds of the object, with associated RGB values, can be used to create the "as-built" 3D model.

After the metric data acquisition, the realization of a parametric model can be developed. The modelling and segmentation of shapes, geometries, and the other building components, by considering the previously acquired metric data, allow the creation of the 3D model.

The level of detail that it is necessary to generate by considering the goal of the work (e.g. quantity and quality of the data to be connected, kind of analysis to be performed, etc.), strongly influences the complexity of the model.

There are different methods to create a parametric model: manual modelling of volumes and shapes, semi-automatic approach, modelling using the cross sections; and automatic extraction of shapes from the point cloud. The last one is not suitable for historical buildings, due to the usual complexity of shapes and geometries (Chiabrando et al, 2017). Indeed, historical buildings combine complex surfaces, distorted areas, ornaments and decorations.

To create an HBIM of an historical building, the possibility to create shapes and surfaces of its main features should be considered: architectural stratigraphic units, and pathologies or degradation of surfaces may be included inside this phase to generate independent volumes if different data and considerations must be connected to them. In this sense it could be possible to extract from point clouds also single architectural components, such as quoin, brick, roof tile and other wall entities.

After this operation, obviously based on the project aim, all these features can be included (using IFC format) into a building entity to create a complete multilevel building.

However, simplification is a necessary step to create an IFC parametric model of an historical building and it is due to the needs to combine the imperfection of the real geometry and the process of modelling shapes from point cloud.
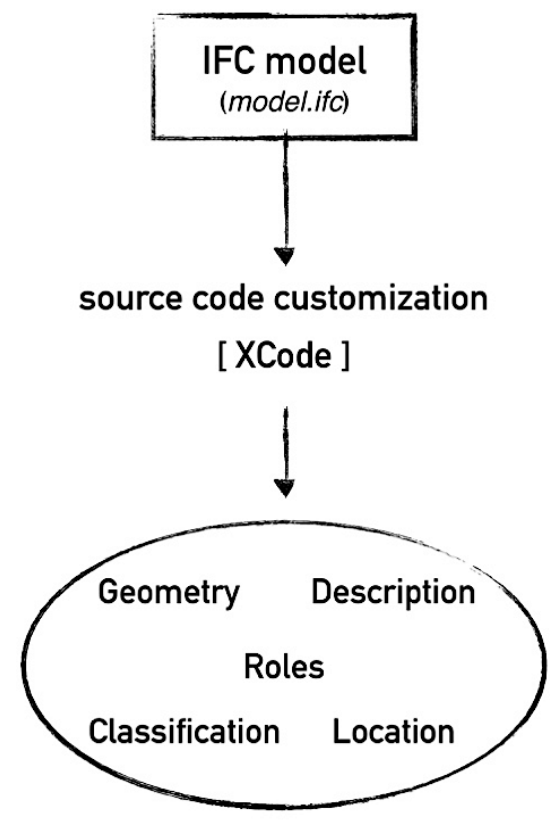

Figure 2. Schema of IFC model customization by using XCode

In addition, the representation of stratigraphic units and other kinds of features and pathologies should be made by creating 3D surfaces and then by defining them as "building element". These elements must be interpreted, represented and then approximated according to the level of detail that the research needs (Lo Turco et al, 2017).

By using XCode, that is an integrated development environment of MacOS (it supports different development languages such as C, C++, Java, AppleScript, Python, Swift), the customization of an IFC file becomes possible. Parameters and properties of an IFC file, such as description, roles, and classifications, can be modified. This approach is useful after the creation of the models to set and fix some IFC parameters and to verify the whole source code of the IFC model.

Although it seems a flexible operation, the modification of IFC source must be made carefully by considering also the integrity of the model, especially whether it is related to other IFC models (backup is strictly recommended).

\section{DBMS FROM IFC DATA}

FreeCAD uses a file format, the so-called "FCStd", to record and collect all data, especially XML documents which contain all steps and properties of the IFC file creation.

To extract a complete data set from an IFC model created with FreeCAD the IFC File Analyser, developed by the National 
Institute of Standards and Technology (NIST) has been tested. This tool generates CSV (comma-separated values spreadsheet) or XLSX (Excel) files from an IFC file. Through this operation all the information concerning IFC file, including building elements, materials, geometry, relationship and other properties, can be extracted. Although CSV is the most used format by considering its flexibility, a better solution could be considered the XLSX format for the creation of worksheets inside a single spreadsheet file, while the CSV exportation allows obtaining different file for each IFC entity. By exporting XLSX file, the attributes instances of an IFC entity are stored in every row inside the worksheet.

CSV and XLSX data extracted form IFC models can be stored and managed inside a relational or spatial DBMS (Database Management System). A simple schema of this proceeding is visible in Fig.4.

\subsection{Relational DBMS}

The creation of a relational database is a fundamental step for an HBIM project to collect and query geometric and alphanumeric data that can be used to design and manage future interventions, to document the present situation, or recreate a previous situation of the object (Lo Turco et al, 2017). Moreover, database benefits can be several, such as backup and data recovery as well as data sharing and integration (Logothetis et al, 2017).

One of the most common DBMS, based on Structured Query Language, is MySQL. In fact, it is the world's most popular open source database, enabling the cost-effective delivery of Web-based and embedded database applications.

In this work MySQL was tested using Sequel Pro (only for MacOS), a simple database management application for working with MySQL databases. It allows creating and managing relational database based essentially on tables that can be investigated through query processes (Fig. 3).

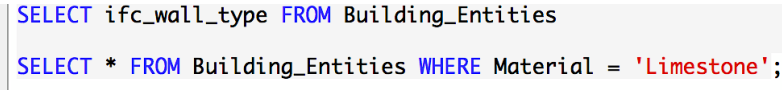

Figure 3. MySQL simple query based on SELECT command

For this purpose, the stratigraphic analysis of an historical architecture or archaeological site and related to IFC surfaces could be investigated through custom-made relational query processes, for example interacting table as "IFC Building" and "stratigraphic lecture". In this sense, the historical evolution (and then the time dimension) is observed and questioned thinking about specific and customizable needs of the research and of final distribution of the data and their relationships.

The complete analysis of a Cultural Heritage context could be carried out by interacting the extracted data form an IFC model (entities, elements, geometry, location, materials, etc.) and other kind of data linked to the object, such as the historical evolution as well as bibliographic resources, archives data, past restorations, images, and so on.

\subsection{Google Documents}

Tables created in Sequel Pro can be exported in CSV or XML format for further management operations. For example, CSV tables can be imported also in Google Sheets (inside Google Documents online package) and then exported in XLSX format, because the importing step modify the CSV structure from comma separated values to a classic table with columns.

In this regards Google Documents could have a dynamic role inside this project. In fact, by using Google Modules, useful for custom-made digital surveys based on alphanumerical and file storage, the automatic compilation of sheets is possible, because this draw up involve the automatic compilation of a Google Sheet and then, after this step, the sheet created can be managed and exported as said before. Indeed, an easy way to perform a starting and simple DBMS is by using Google Modules and then a customized survey to fill out, which creates automatically a Google Sheet that could be imported as a table inside a SQL based database to enrich and interrogate it.

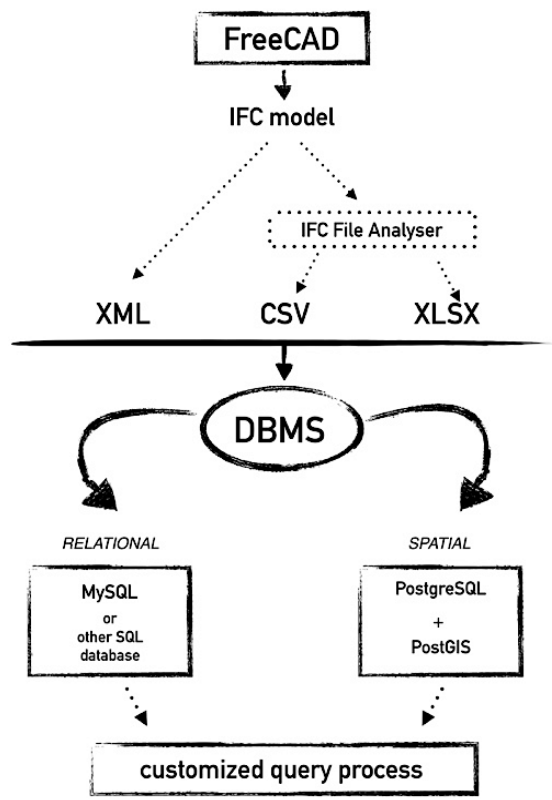

Figure 4. From IFC model created with FreeCAD to DBMS and query process

\subsection{Spatial DBMS}

Up to now, the spatial DBMS was not tested. However, for further analyses, the creation of a spatial database will be considered too, because it becomes very important to manage geometrical data, location of elements and topological data through their spatial relations.

In this sense, the most suitable open source solution (able to be connected to FreeCAD) is the utilization of PostGIS plugin inside PostgreSQL database. This plugin allows managing geographic data for the PostgreSQL object-relational database (Logothetis et al, 2017). The possibility to include in this project PostgreSQL and PostGIS plugin ensures to manage the collected data into a 3D GIS system, useful for geographic and spatial analyses.

\section{USABILITY: WEB SERVERS AND VIEWERS}

IFC models, data sheets and other kind of data related to the studied object must be shared in the best way possible.

The usability and querying process of data is a fundamental requirement of an HBIM project.

Through the utilization of any kind of BIM servers, such as BIM Server or IFC Web Server, BIM can be considered as an open service for the community.

Therefore, BIM servers could allow to work into an environment where different professional figures can contribute to collect others information and data referred to a specific context.

The connection of different data to 3D/IFC models allow therefore a multidisciplinary, collaborative and open approach. Moreover, BIM servers have a fundamental importance to 
collect and share final data to other users (Logothetis et al, 2016).

The idea to transform a project into a sharable project open to the interested community requires a strong and complete documentation of the whole HBIM creation process. This kind of "meta-documentation" is essential to guarantee the correct interpretation of the adopted solutions and the coherent input of other data by different users.

\subsection{BIM Server}

BIM Server is an open source standalone tool that allow sharing BIM projects. This tool can be used with an online server or in local (localhost).

To install BIM Server is necessary to have Java package installed inside the platform. FreeCAD allows the integration with BIM server. To do that it is necessary to install $J_{\text {son }}$ and Requests Phyton modules.

BIM Server essentially allows to visualize and analyse IFC 3D models and to make revisions, comments and to upload other kind of data inside the project.

In addition to these features, BIM Server allows (due to some opens source plugins) to query, merge, filter IFC models, and to read and write IFC files in real time.

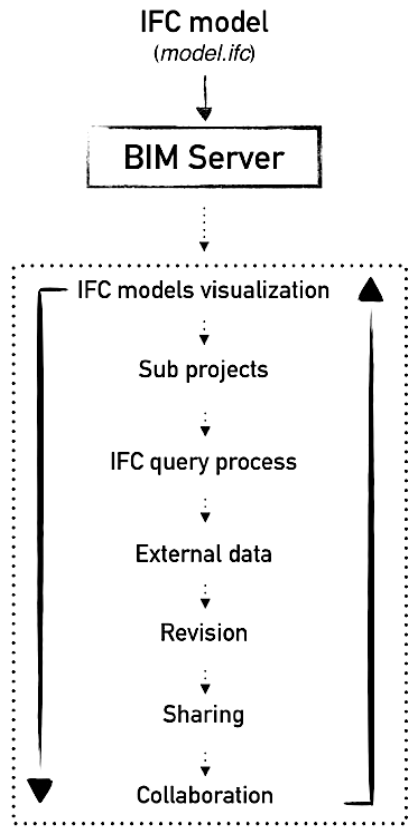

Figure 5. Example of a basic workflow by using BIM Server

6.1.1 BIM Server customization: Inside BIM Server, IFC structure and component objects created using FreeCAD, are classified as BuildingElementProxy: this is a basic element in an architectural or engineering BIM project, but not properly fitting to a Cultural Heritage object.

However, the modification of this kind of definition is possible by re-writing the schema inside the IFC2X3TC1.exp file or IFC4.exp file of BIM Server (e.g. the file where the IFC object definitions are expressed).

Moreover, to ensure right modifications, the definitions must be fixed by modifying (using XCode) directly the IFC model file (as seen in the 4.0 section) according to the IFC2X3 definitions schema.

A quick and clear modification (useful for historical buildings) can be done replacing "BuildingElementProxy" with the standard and generic definition "Element" inside the IFC model file (see Fig. 7 and 8).

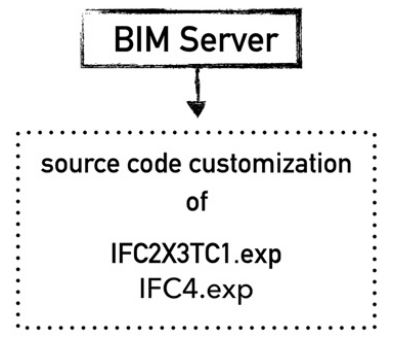

Figure 6. BIM Server file customization

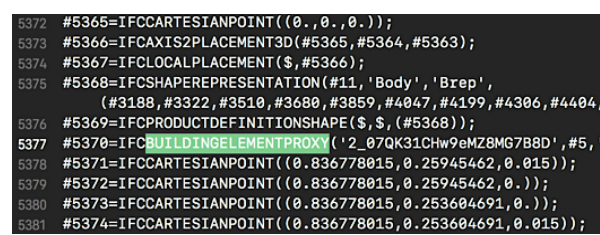

Figure 7. IFC entity of line 5377 classified as "ifcbuildingelementproxy"

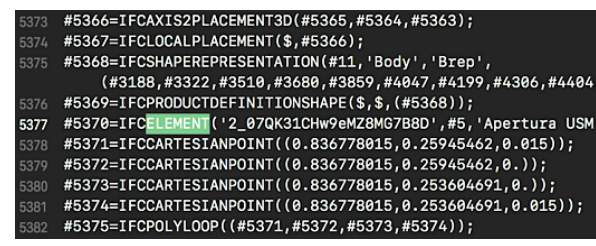

Figure 8. "ifcbuildingelementproxy" changed with "element"

This kind of definition may be now visible into BIMViews, on the graphic interface of BIM Server, in particular on types menu (see Fig. 11).

6.1.2 Querying an IFC model using BIM Server: Inside BIM Server the investigation of IFC models is possible simply by querying them. In fact, the installation of different query engines (as plugin), such as BimQL and Java Query Engine Language, is also possible. The last one is based on the eclipse modelling framework and Oracle Berkeley DB, a basic database inside BIM Server (Ghang et al., 2014).

Figure 11 shows a Java Query Engine Language script to query a wall selection of a masonry of an historical building.

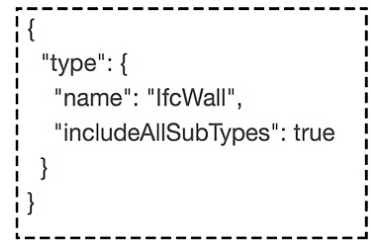

Figure 9. Java Query Engine Language script to query a wall selection form IFC model including all sub types.

This kind of simple query can be modified, according to building entities and classification.

Moreover, more complex queries (also relational ones) can be write to investigate all the properties of the IFC model.

\subsection{Viewers and checkers}

BIM viewers allow to open and visualize 3D IFC file of a BIM project. Software like Solibri Model Viewer (www.solibri.com), are essentially developed to allow the final distribution of the BIM project to generic users who want just to enquire it. 

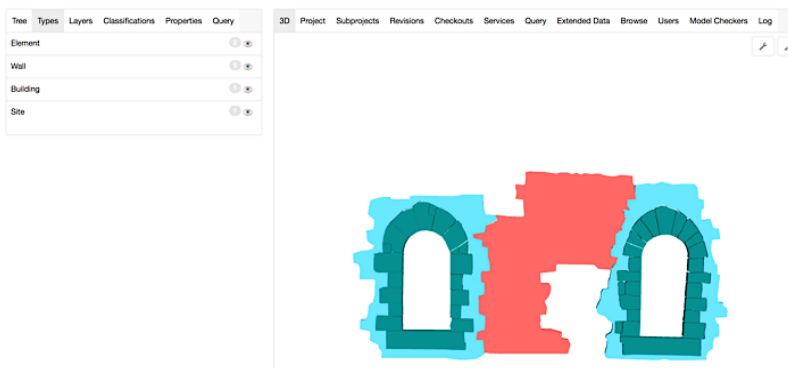

Figure 10. BIM Server. Example of masonry with stratigraphic units. Stone windows (wall cavity) are classified as "element"

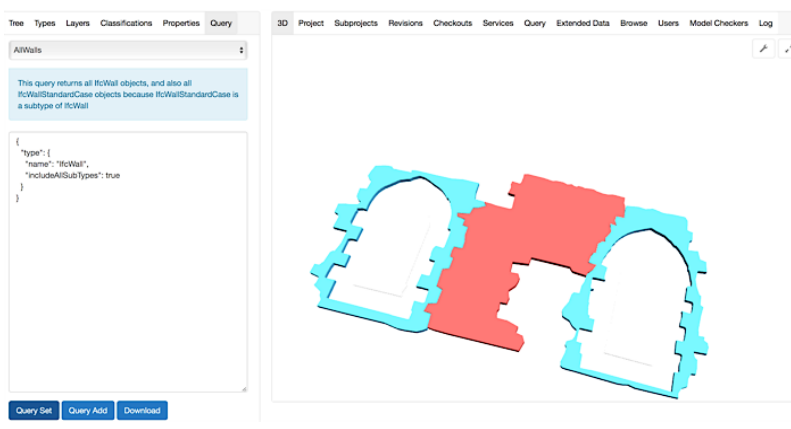

Figure 11. BIM Server. Example of a simple query to show only wall (ifcwall) elements of the masonry

This commercial tool, available in free and professional version, permits the visualization of the entire model tree (e.g. default site, default building and all IFC classification and entities).

All IFC properties, such as identification, location, quantities, relations, classification, hyperlinks, can be visualized, checked and managed.

Apstex using open source libraries such as Java, develops another BIM viewer: IFC Java Viewer. As the previous described solution, this software allows to communicate and explore IFC models and their properties. Inside this viewer (in Step File View) the entire IFC source code is visible, and it is useful to search, select and copy all the 3D model features.

Solibri Company developed a model checker and an IFC optimizer.

Briefly, Solibri Model Checker is a rules-based checking software for compliance and validation of all the objects of a model, while Solibri IFC Optimizer allows the optimization of IFC schema, by reducing, as an example, large IFC file sizes for viewing or distributing.

Viewers and checkers are very simple tools that do not allow, until now, the querying of the model.

Therefore, the use of viewers and checkers permits the interactive navigation of IFC models and this use becomes a common reference frame devoted to team's members (Logothetis et al, 2016).

\section{CONCLUSIONS}

BIM solutions permit to do better managements of architectural design and building processes. Indeed, it is considered the best way to collect and link together different data with a unique 3D parametric model.

However, the use of this approach for historical architecture and for Cultural Heritage, in general, is still an ongoing research field and an interesting method to do an interdisciplinary research (Chiabrando et al, 2017).
The integration of stratigraphy and then the stratigraphic units (also pathologies) of an historical architecture or an archaeological site is a critical point to be managed inside a BIM system. To include and manage other kinds of data related to surfaces and shapes not directly connected with a common BIM thought and then without a specific architectural or structural classification is always a difficult task (Chiabrando et al, 2017).

However, as seen before, this kind of data may be represented and classified graphically by customizing the materials database and by modifying the source code of architectural or environmental components of a parametric open source CAD, such as FreeCAD.

Moreover, the documentation and the interpretation of these elements can be carry out by using relational and spatial database connected with the IFC model, which has to be investigated through some query processes in order to analyse it in the best possible way.

Time is a fundamental feature inside an HBIM for historical architecture or archaeology; therefore, the integration of stratigraphic analysis make sense. By adopting this strategy, the evolution (4D) of the analysed object is correctly carried out, accordingly to the study and the communication of the historical evolution through different steps as: visualize, read and query.

The work presented might be considered as a starting point for a future creation of a complete HBIM open source solution that could be adapted to others Cultural Heritage researches and analysis.

To adapt geomatics techniques and solutions to the research field and not the opposite is the correct approach that researchers must pursue.

The automatization of some steps will help the diffusion of the approach, but it must be considered that the HBIM creation is essentially an intelligent phase. The big variety of Cultural Heritage assets and the huge number of variables that influence each kind of investigation of them do not allow to look for a unique solution for all the possible cases, but to define a unique intelligent approach to the problems.

The aim of this project proposal is the future creation of a dynamic and open system, probably based on FOSS solutions that could be adopted without restrictions and used as a specific Cultural Heritage research requires.

\section{ACKNOWLEDGMENTS}

This work was supported and funded by the GAMHer project (Geomatics data Acquisition and Management for landscape and built Heritage in a European perspective), a 3 years project financed under the Italian PRIN 2015 framework (Progetti di Ricerca di Rilevante Interesse Nazionale).

\section{REFERENCES}

Barazzetti, L., 2016. Parametric as-built model generation of complex shapes from point clouds. In: Advanced Engineering Informatics, 30 (2016) 298-311

Chiabrando, F., Lo Turco, M., Rinaudo, F., 2017. Modeling the decay in an HBIM starting from 3D point clouds. A followed approach for Cultural Heritage knowledge. In: The International Archives of the Photogrammetry, Remote Sensing and Spatial Information Sciences, Ottawa, Canada, Vol. XLII2/W5, pp.605-612

Ghang Lee, Jiyong Jeong, Jongsung Won, Chiyon Cho, Seokjoon You, Sungil Ham, and Hoonsig Kang. 2014. Query 
Performance of the IFC Model Server Using an ObjectRelational Database Approach and a Traditional Relational Database Approach. In: Journal of Computing Civil Engineering, 2014, 28(2): 210-222

ISO/TC 184/SC 4. (1994). "Industrial automation systems and integration - Product data representation and exchange-Part 11: Description methods: The EXPRESS language reference manual." ISO 10303-11:1994, Geneva.

Lo Turco, M., Mattone, M., Rinaudo, F., 2017. Metric survey and BIM technologies to record decay conditions. In: The International Archives of the Photogrammetry, Remote Sensing and Spatial Information Sciences. Florence, Italy, Vol. XLII5/W1, 2017 Geomatics \& Restoration - Conservation of Cultural Heritage in the Digital Era, 22-24 May 2017, pp.261268

Logothetis, S., Karachaliou, E., Stylianidis, E., From OSS CAD to BIM for Cultural Heritage digital representation. In: The International Archives of the Photogrammetry, Remote Sensing and Spatial Information Sciences. Nafplio, Greece, Vol. XLII2/W3, 2017 3D Virtual Reconstruction and Visualization of Complex Architectures, 1-3 March 2017

Logothetis, S., Valari, E., Karachaliou, E., Stylianidis, E., 2017. Spatial DMBS architecture for a free and open source BIM, In: The International Archives of the Photogrammetry, Remote Sensing and Spatial Information Sciences, Ottawa, Canada, Vol. XLII-2/W5, pp.467-473

Logothetis, S., Stylianidis, E., 2016. BIM Open Source Software (OSS) for the documentation of Cultural Heritage. In: Virtual Archaeology Review, 7(15): 28-35

Macher, H., Landes, T., Grussenmeyer, P., 2017. From Point Clouds to Building Information Models: 3D Semi-Automatic Reconstruction of Indoors of Existing Buildings. In: Applied Sciences 2017, 7, 1030

Myers, D., Dalgity, A., \& Avramides, I., 2016. The Arches heritage inventory and management system: a platform for the heritage field. In: Journal of Cultural Heritage Management and Sustainable Development, 6 (2), 213-224. 